\title{
THE ROLE OF SUB-DISCIPLINE CHOICE IN WOMEN'S ENROLLMENT AND SUCCESS WITHIN CANADIAN UNDERGRADUATE ENGINEERING PROGRAMS.
}

\author{
Juliette Sweeney \\ OISE, Engineering Education Collaboration Program, University of Toronto \\ juliette.sweeney@mail.utoronto.ca
}

\begin{abstract}
In Canada, the proportion of female students in engineering is considerably lower than the proportion of female students in higher education. Using Tinto's (1993) theories concerning social and academic integration, this study investigated the relationships between the proportion of female undergraduate engineering students, and the proportion of female faculty, and departmental lead faculty. Using descriptive statistics, the study established that distinct and persistent differences exist in the proportions of female enrolment among schools and among sub-disciplines. This paper addresses a gap in the literature concerning the impact of sub-discipline choice on women's engagement and success within undergraduate engineering programs in Canada. The proportion of female students was found to vary considerably across the sub-disciplines, from $48 \%$ in biosystems to $15 \%$ in software engineering [14]. The paper will present female sub-discipline enrolment trends over time and discuss the impact of sub-discipline choice and institutional factors on female students' successful academic and social integration within Canadian engineering schools.
\end{abstract}

Keywords: diversity, sub-discipline, gender, faculty, undergraduate, education, success, STEM.

\section{INTRODUCTION}

While the number of women in Science, Technology, Engineering and Mathematics (STEM) education and careers has increased over time, STEM has not seen the same equity gains as those achieved in other science-based fields such as medicine. In 1995 Canadian medical schools admitted equal numbers of women and men for the first time [2]. However, over two decades later, in 2017, the proportion of female students in Canadian university engineering undergraduate programs was only $22 \%$, with the sub-discipline of software engineering languishing at $15 \%[14]$.

\subsection{Literature Review:}

Most researchers concerned with female representation within STEM would agree that the problem is complex and various solutions are required to increase representation. Multiple factors cause women to exit a STEM career path at many points [4]. Women's decisions to participate in STEM are influenced by societal, institutional, and individual factors that may act alone or in combination, and potentially have a cohesive or contradictory impact.

Research has found that female engineers have adopted different strategies to build a strong sense of professional identity and succeed in engineering. Women need to work harder to gain technical acceptance and may block or deflect offensive male behaviour by using humour. Women need to leverage the interests of others to ensure their accomplishments are recognized, and that their professional identity as an engineer is respected [20].

Many researchers note the impact women's socialization has had on career choice and professional identity selection. Godec [17] frames women's socialization as critical in identity formation, noting that women's socialization has traditionally promoted caring for others and may make it easier for a woman to explain a career choice in health services, rather than engineering. Research from the US claims that engineering schools have been found to contain a gendered professional identity process [10], which results in gendered career options. Other research finds many engineering students [22] and faculty [5] hold gendered notions of what it means to be an engineer, while other authors have argued that engineering schools contain chilly climates, with social and academic isolation being common experiences for female engineering students [10], [28].

Examining how career expectations influence engineering students, female students were found to demonstrate a higher requirement to affect positive social change as part of their professional identity [2], [18]. Both engineering schools and the profession have been slow to articulate the ability of engineering to improve society and their environment [7]. Students looking to achieve social change within their profession may select the subdiscipline of biosystems because of its perceived proximity 
to the medical field, which is viewed as a profession that improves society [17]. This can be contrasted to the field of software engineering, which is often perceived as a commercial sector, unlikely to improve society or address social change [16].

Even seemingly positive changes to the curricula influenced by industry have been found to have negative effects in terms of meritocracy. Many schools have incorporated team projects into their curricula to provide students with an experience similar to that of the workplace, and to develop collaborative team building skills [27]. However, students, having experienced a rigorous sorting process to get into the engineering program, and believing that such a sorting process is "right" because it was based on "merit", apply their own sorting process to team member selection, quickly discarding those they deem to be less technically "fit" than themselves. Female students were more likely to be discarded from teams, or awarded lesser administrative or management roles, that conformed to gender stereotypes, and to be excluded from design or building activities, considered high status within an engineering context [27]. The young women, while conscious of their mistreatment, evaded questioning the meritocratic ethos of engineering or complaining to faculty about these instances. Similar findings to this US study were found in Canadian universities [24] which discovered that both female and male students were reluctant to report occurrences of discrimination due to fear of retaliation from fellow students.

\subsection{Problem Definition}

The lack of women in Canadian engineering programs is deeply concerning from economic and social perspectives. From an economic standpoint, demand for engineers in Canada continues to outstrip supply in most sub-disciplines [11] and evidence that diverse engineering teams build better products [26] is repeatedly stressed by leaders in the field. From a social perspective, it should be acknowledged the homogeneity of teams has exacerbated design flaws [25], [6]. Increasing women's, and other minorities, engagement in engineering is critical because engineers design and build the tools we use, the environment we live in and the ways in which we learn. If technology-driven solutions are designed and built by teams comprised mainly of men, both the analysis of problems and the design of solutions may exclude nonmale perspectives.

As an example, the initial design of air bags was intended to increase driver and passenger safety, unfortunately, "first-generation air bags were designed to protect an unbelted adult male at the 50th percentile of body height and weight in a severe frontal crash" [25] p. 744. The result was a design that did not take into account smaller, lighter body frames, which resulted in injuries or death for many women, children, and smaller men until this flaw was acknowledged and corrected [25].

In contemporary technology, a recent study has shown that facial recognition software misrecognizes women at a much higher rate than men. In a 2018 study of top four best selling commercial software recognition packages, error rates for light-skinned men were reported as low as $0.8 \%$, whereas error rates for darker-skinned women were documented as high as $38 \%$ [6]. This study notes that such results are potentially reproducing representation bias from internet images which are used to 'train' the artificial intelligence used to drive the recognition process. Air-bags were not designed to deliberately hurt women, children and smaller men, neither was facial recognition technology designed to produce sexist and racist results. These design flaws were neither deliberate nor are they unsolvable. However, both examples illustrate how homogenous teams, with similar worldviews, can overlook or exacerbate design flaws for sections of the population that are not well represented on their teams.

Women have also faced longstanding discrimination in engineering, experiencing persistent wage and opportunity gaps based on gender. The software industry in particular suffers from a lack of women in senior positions and gender diversity [19] and factors limiting equity in this field need to be identified and addressed.

\subsection{Motivation for the paper}

To address gender representation in the engineering sectors workforce, it is useful to understand how gender representation plays out within the various sub-disciplines in engineering schools. Understanding how the proportions of female students in Canadian engineering sub-disciplines have changed over time will expand the literature and research that addresses sub-discipline choices made by women in engineering programs. While data is readily available detailing both enrolment and graduation rates for women in the various engineering subdisciplines in Canadian universities [14], analysis of the proportions of female students within the Canadian subdisciplines at a national and institutional level is lacking. A better understanding of gender differences among subdisciplines has the potential to help provincial governments and higher education institutions to build policies aimed at increasing gender diversity in the field.

\subsection{Methodology}

Enrolment and graduation data available from Engineers Canada for the years 2008-2017 provided the base data set. The aim of the analysis was to determine what the current proportions of student and faculty are women, and what historical trends, if any, are apparent in the data. The data analysed covered 10 academic years and the 20 largest engineering schools in Canada. These 
schools represent over $80 \%$ of the total undergraduate engineering population in Canada.

Descriptive statistics were used to establish the current proportion of women in both student and faculty populations. Given that data analysed in this study covered 10 academic years and 20 different schools and involved thousands of data points, a quantitative approach using descriptive statistics facilitated the presentation of a large amount in summary format. The study addresses the lack of longitudinal, quantitative research on the low proportions of women in student and faculty populations within Canadian engineering schools, and comparisons of institutional results in this area. This research calculated proportions for both female students and faculty at the national and institutional levels over the period 2008-2017 for engineering programs as a whole, and each subdiscipline with a particular focus on biosystems and software engineering. Growth rates over the ten years were then computed for students for engineering programs as a whole, and the sub-disciplines of biosystems and software engineering.

While all data used by this research are publicly available through Engineers Canada websites, no formal vetting procedure exist to ensure that engineering schools report their data and use nomenclature consistently when providing their enrolment data to Engineers Canada. However, given the visibility and use of these data within industry and academia, it was assumed that engineering schools are motivated to ensure data quality in their reporting to Engineers Canada. While data from Engineers Canada contained student data at the sub-discipline, faculty data was only available at the institutional level. Data on departmental lead faculty was derived from institutional websites over the period January-June 2017 and as such represent a snapshot in time.

Data on the gender of students and faculty was supplied by engineering schools as portion of their enrolment data that is supplied annually to Engineers Canada. Data on the gender of departmental lead faculty was assumed based on data available on institutional websites. This study reports gender on binary basis, but does acknowledge that gender is a complex construct. Due to scope limitations, this research was unable to address the various forms gender can assume and the elements of intersectionality that often compound the negative impacts of gendered processes, such as race and class.

\subsection{Conceptual Model}

Tinto's Model of Institutional Departure [30] from 1993 posits that there are two systems within higher education institutions that students need to successfully integrate with in order to succeed: academic and social. The model suggests that students enter colleges and universities with goals and commitments, and that these are consistently revised to reflect that the level of academic and social integration achieved by the student within the institution. These modified goals will impact the student's decision to leave or stay in the institution [1]. As shown in figure 1, various elements were placed in Tinto's model to suggest how female students may attempt to achieve academic and social integration by their actions, including the subdisciplines they select.

This research applies Tinto's model to propose that female role models, exemplified by faculty and academic leaders who were women, may improve female students' ability to integrate academically and socially into their institutional environments. Female role models could normalize the presence of women within the engineering environment by both their presence and performance. Successful female role models will also reduce negative stereotype threat, defined as the fear of being identified with a negative stereotype leading to lower performance [23].

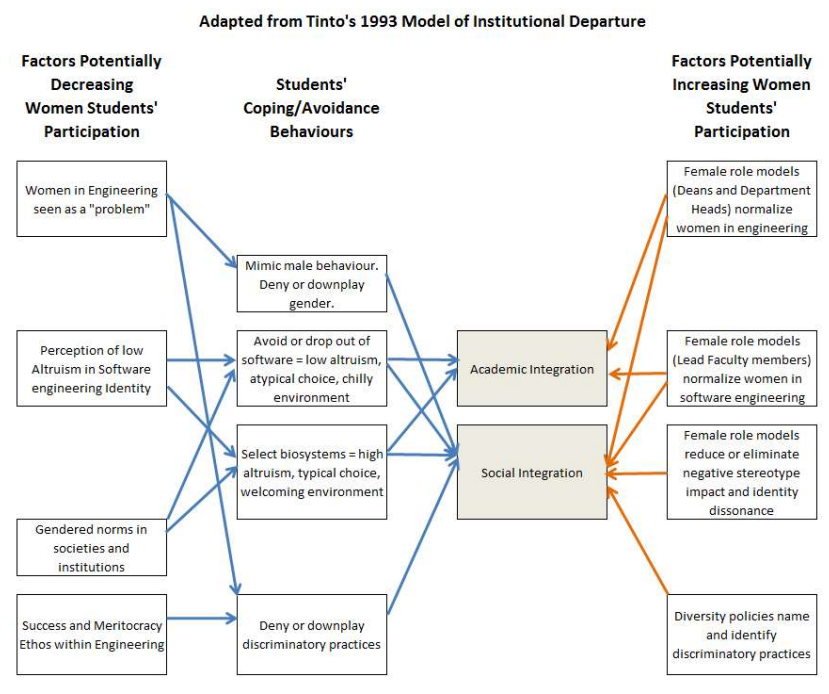

Figure 1. Map of Factors influencing Female Students' Academic and Social Integration, and by extension, participation. Adopted from Tinto's 1993 Model of Institutional Departure.

\section{FINDINGS}

\subsection{Proportion of Women Students in Engineering}

During this research national proportions of female students and faculty were computed for the engineering discipline as a whole, and then the proportions of female students were also calculated for all sub-disciplines with a particular focus on biosystems and software engineering.

While the proportion of female students in engineering is growing, the rate of change is slow as shown in Figure 2. In 1991, the proportion of female students in Canadian engineering programs was $16.1 \%$ and over the next 26 years only rose by $5.7 \%$ to reach $21.8 \%$ by 2017 . It is 
important to note that while many countries share Canada's challenges in this area, others do not. The low proportions of female students graduating in Canada are similar to certain countries in the Americas and Europe, but other geographies report much higher proportions of female graduates. In 2012 the proportion of women graduates in Canada was $20 \%$ [14]; in the US, $18.5 \%$; the UK, $22.2 \%$; and in France $25.6 \%$. At the higher end of the spectrum Poland's engineering 2012 graduate class cohort contained $31.6 \%$ women; Malaysia's, 38.7\%; and Oman's, 52.7\% [31]. It appears that the proportion of engineering graduates who are women differs by geography and culture.

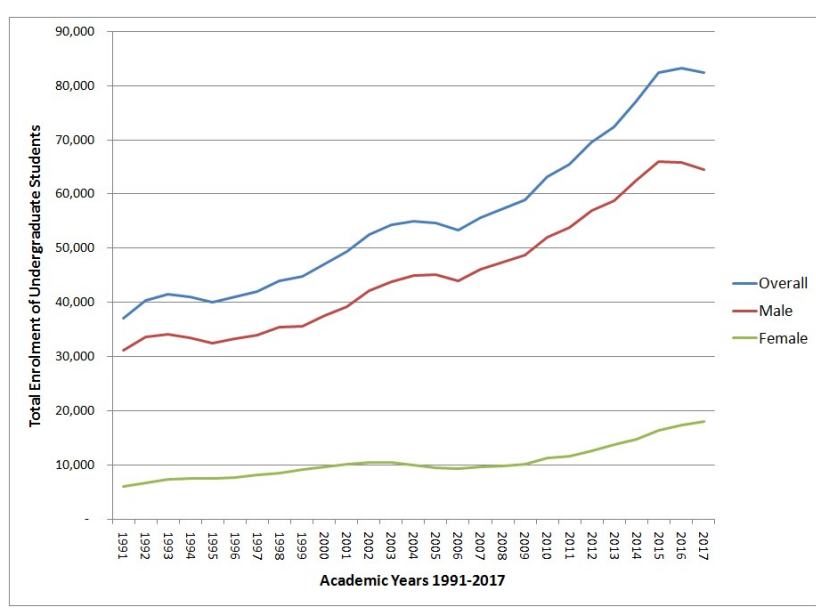

Figure 2. Slow annual growth in female undergraduate engineering enrolment in Canada: 1991 to 2017.

Figure 2 shows slow overall enrolment growth in engineering from 1991 to 2017, with negative growth seen for both female and male students in some years; however, overall growth for the entire period was positive for both genders. The number of engineering students that are women is still significantly lower than those who are men with the ratio of male to female students in 2017 being more than $4: 1$.

\subsection{Proportion of Women Students in the Sub- disciplines}

Moving to examine enrolment rates at the subdiscipline level, it became apparent that the proportion of women students and their enrolment growth rate varied significantly across the sub-discipline specialist areas. While taking a common first year before selecting a subdiscipline is becoming more popular, most undergraduate students still elect to enrol in a specific sub-discipline directly from high school. The proportion of students who are women in the various sub-disciplines varies considerably. Contrasting biosystems with software engineering it was calculated that the proportion of women in the sub-discipline of biosystems approached $50 \%$ while the proportion of women in software engineering continued to lag at less than $15 \%$. Trend analysis indicated gendered enrolment in the sub-disciplines was distinct and relatively consistent over time. The top four subdisciplines in 2004, with the highest percentages of their total population being women, were biosystems, environmental, chemical and geological, and all four remained in the top quartile in 2017. At the other end of the scale, the four lowest sub-disciplines in 2004, electrical, computer, software and mechanical, still resided at the lowest quartile by 2017 .

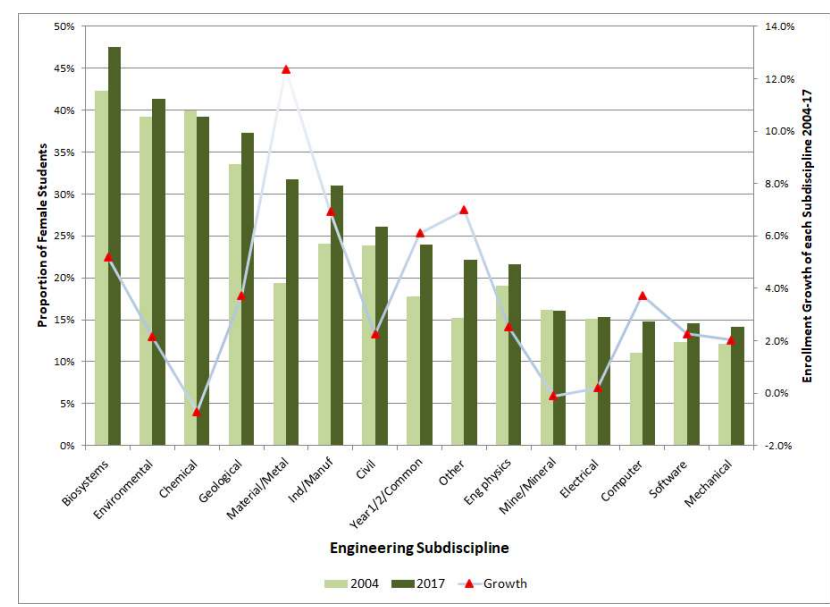

Figure 3. Percentage of women students in each sub-discipline: 2004 vs. 2017.

Figure 3 shows that over the period 2004-2017, all subdisciplines increased their proportion of female students, except for mining/mineral engineering, which declined slightly. Average growth in female enrolment across most sub-disciplines was modest, with the overall proportion of women increasing by just over $4 \%$ over 13 years. Only two sub-disciplines experienced an increase in enrolment rates that exceeded 5\%: biosystems and industrialmanufacturing; and only one sub-discipline, materialmetallurgical, had a rate that surpassed $10 \%$ over this time period.

The sub-disciplines with the highest proportion of female students were not the same as the sub-disciplines with the largest number of female students. From 2004 to 2017 , four subdisciplines usually accounted for over $70 \%$ of female enrolment: civil, mechanical, chemical and electrical. The proportion of women in chemical (39\%) is relatively high, in civil (26\%) close to average, and in electrical $(15 \%)$ and mechanical $(14 \%)$ is very low. Mechanical engineering dominated overall enrolment during 2004-17, always accounting for at least $21 \%$ of total enrolment, but consistently displayed a low proportion of women students. Given its share of enrolment, the subdiscipline of mechanical engineering has a strong 
impact on the average proportion of women students overall, and potentially the culture within undergraduate engineering programs.

\subsection{Women students in Biosystems and Software Engineering}

Accredited biosystems and software engineering programs are relatively new additions to engineering departments in Canadian higher education. Guelph University was the first school to introduce a biological engineering program in 1972 and software engineering was first offered at McMaster University, Ottawa University, and Western University in 2001 [14] Appendix B, p. 77. At the national level, enrolment for both sub-disciplines has grown rapidly over the period 2004-2017 with enrolment in biosystems growing by $281 \%$ and software engineering growing at $241 \%$. The growth rates for both sub-disciplines are more than six times greater than the overall average enrolment growth of $50 \%$. The growth of female enrolment in both sub-disciplines also exceeds that of men by a significant margin, $81 \%$ for biosystems and $71 \%$ for software engineering.

While female enrolment grew at similar rates for both sub-disciplines, the proportion of students that were women in the two sub-disciplines differed significantly. The proportion of women in biosystems was always greater than the overall average proportion of women in engineering by more than 7\%. During 2004-17 the proportion of female students in biosystems ranged from 33 to $44 \%$ with software engineering displaying a range between 10 to $15 \%$ during the same period.

While these sub-disciplines have experienced rapid growth they still capture a relatively small portion of the total engineering student population. Biosystems has increased in popularity with both women and men. In 2017 , biosystems attracted $4.9 \%$ of female engineering students and $1.5 \%$ of male students compared to 2004 when it only attracted $2.1 \%$ of the female students and $0.6 \%$ of male students. Software engineering has decreased in popularity with both genders. In 2017, software engineering was chosen by $4.1 \%$ of female students and $6.6 \%$ of male students; with its share of the total student population decreasing if compared to 2004 when this subdiscipline was chosen by $12.7 \%$ of all female students and $12.5 \%$ of all male students.

In summary, both sub-disciplines have demonstrated strong enrolment growth, especially for students who are women. However, during the time period examined, biosystems exhibited consistently high proportions of female students while software engineering exhibited consistently low proportions of female students. This difference could be explained within Tinto's model as the close to equal numbers of women and men in biosystems programs offers aspiring engineers who are women an environment where women's presence is normalized and more opportunities for social and academic integration exist. While both sub-disciplines continue to grow at a rapid pace, it is important to note that in 2017 their combined enrolment only represents $9 \%$ of all female students and $8 \%$ of male students. The rapid growth rate of these sub-disciplines echoes the growing importance and resource demands of biotechnology and software engineering not only within Canadian society, but also at a global level.

\subsection{Women Faculty in Engineering}

Data on engineering faculty in Canada, for women and men, were available for 2010-2017. Data was available at the individual school or college level, but not at the subdiscipline level. In 2017 the proportion of engineering faculty who were women was $15.9 \%$ which represented a $2.3 \%$ increase from 2010 .

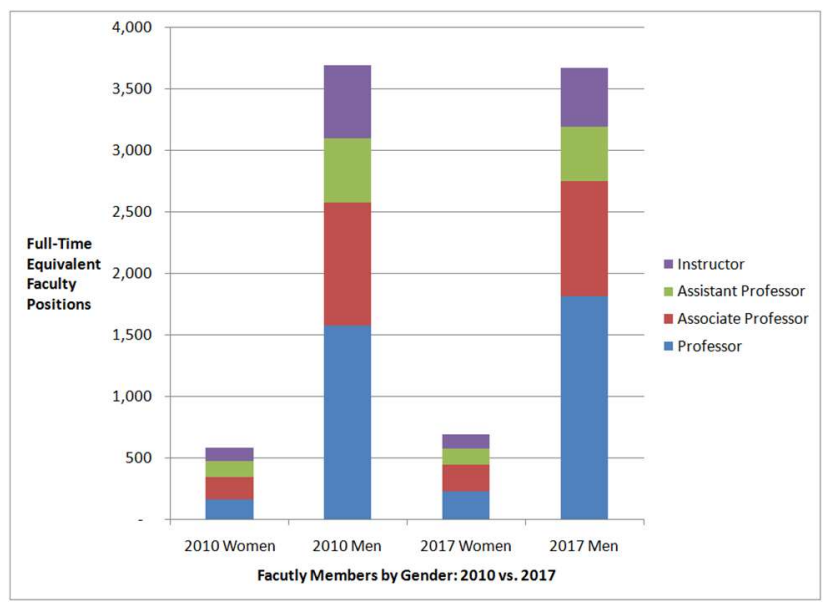

Figure 4. Engineering Faculty positions by gender, 2010 vs. 2017

As shown in Figure 4, faculty numbers were reported at four different levels: Professor, Associate Professor, Assistant Professor, and Instructor. Female faculty tended to cluster at the lower ranks. In 2017 the proportion of women at the Professor level was $12.8 \%$, at the Associate Professor level 22.7\%, the Assistant Professor level 30.3\% and at the Instructor level $24.2 \%$. The proportion of women in engineering faculties in Canada is low compared to all other disciplines. In 2017 women accounted for nearly $39.6 \%$ of the faculty population in Canada, for $27.6 \%$ of professors, $43.0 \%$ of associate professors, $48.5 \%$ of assistant professors, and $53.7 \%$ of instructors [29].

In summary, the proportion of women in engineering faculties is low, $15.9 \%$ compared to the national proportion of all women faculty in Canada of $48.5 \%$ and representation of women is disproportionate within the lower ranks with over $36 \%$ of female faculty being instructors or assistant professors compare to less than $26 \%$ of male faculty being in these positions. The growth of 
women as a percentage of the engineering faculty population is very slow, at $2.3 \%$ over seven years.

\subsection{Women Lead Faculty at the Departmental Level}

The number of female department heads identified in this research represents a particular moment in time, the academic year 2017-2018. Nomenclature for lead faculty members at the schools included in the study varied considerably across schools; as a result, faculty members were selected who appeared to be the most senior within the sub-discipline based on positions title and any visible organizational hierarchy available on the institutional website. In the sub-discipline of biosystems engineering two female lead faculty members were identified. Within the sub-discipline of software engineering no female department heads were identified. With such low representation of women at the leadership level, and the variability of titles for these leadership positions, it was not possible to claim any relationship between female leadership and the proportion of female students within the sub-discipline. It does appear that the higher number of female students in biosystems is reflected in the higher number of lead faculty members for that sub-discipline, but the low levels of female faculty is reflected in the low numbers of women at the departmental leadership level for these two sub-disciplines.

\section{DISCUSSION}

\subsection{The Proportion of Women Students}

While other studies have noted higher female student representation rates within specific engineering subdisciplines in the US [22] and Sweden [15], this study addresses the lack of research in a Canadian context. While researchers have pointed to gendered processes within engineering schools [10], the distinct differences between enrolment rates driving the proportion of female students in sub-disciplines indicate that gendered processes influencing sub-discipline choice also exist prior to university.

An alternate explanation for the different proportions of female students in the sub-disciplines could be that gendered processes are absent or less prevalent in some sub-disciplines such as biosystems. It could also be that these gendered processes may come into play after common first year programs to channel more women toward sub-disciplines such as biosystems and less toward sub-disciplines similar to software engineering. Biosystems proximity to the health care field may also make it an easier career choice for women to justify to others, in terms of conforming to gender typical stereotypes that women are more caring than men [17].
Software engineering' proximity to the IT sector could also cause this sub-discipline to be associated with work environments that lack support for women at best [20], and are toxic at worst [19]. As results from this research in Canada echo similar results reported in the US [22] and Sweden [15], cultural or societal influences common to all three countries could also be at play in this area.

While cultural or social influences experienced prior to post-secondary schooling will influence sub-discipline choice, this paper focuses on how Tinto's model applies to experiences students have within higher education institutions. The results from this research suggest that female students are achieving a higher level of academic and social integration, as defined within Tinto's model, within biosystems programs than they are within software engineering programs. The large proportion of female students within biosystems could be causing a virtuous cycle of increasing female enrolment. Female students could be enrolling in biosystems as they expect a higher level of social integration due to the higher number of women already in the program. A higher level of social integration could lead to a higher level of academic integration, community integration and success, as all these factors would reduce the social isolation and lack of peer support for women in STEM programs has been linked with lower performance [10], [4].

In contrast, the female students in software engineering may be more likely to face social isolation and lack of peer support as a result the low numbers of women in those programs [10], [4]. Female students in software engineering also may face increased negative stereotype threats because of the low percentage of women in the field and media portrayal of the industry which is notable for its lack of female role models [19].

The fact that there was an impact on sub-discipline rates at the institution level, that at some schools all subdisciplines were higher than the national averages, whereas at other institutions they were noticeably lower, also indicates that institutional actions and environments do make a different to the proportion of women students. This finding indicates that the actions of institutions can make a difference in this area, if diversity within the student body is made an organizational priority. This finding is consistent with Tinto's theories on academic and social integration where he places significant emphasis on the responsibility of institutions to ensure that their policies and environments are welcoming and supportive of all students [30]. Investigating and documenting best practices at the institutions, and within the sub-disciplines, that have consistently higher proportions of women would be a fruitful course for future studies.

\subsection{The Proportion of Women Faculty in Engineering}

The results from Holman et al.'s research [21] assumes if a population within a discipline is changing slowly, it 
will continue that slow rate of change. However, other researchers have argued that social norms can change relatively quickly once a critical mass of the population has been convinced to accept a new position [11]. These authors note that the percentage considered critical mass is context sensitive with linguistic conventions requiring only $10 \%$ of a dedicated minority to overturn conventions, but other research on gender conventions note that up to $40 \%$ of the population may be required to overturn conventions [11]. While it does appear that some sub-disciplines have approached these thresholds for the proportions of female students, many have not.

\subsection{Limitations of the Study}

As noted previously, Engineers Canada reports subdiscipline data by certified program, which may not reflect all the activity with a particular sub-discipline at any school. For example, a school may offer software programming concentrations within its electrical engineering program. As a result, students enrolled in these software engineering concentrations will be reported under the electrical engineering sub-discipline. It should also be noted that while all the data used by this research is publicly available through Engineers Canada websites, no formal vetting procedure ensures all schools are report their data and use nomenclature consistently. This could potentially result in data anomalies being present in the datasets analysed for this research. However, as the Engineers Canada enrolment reports are regularly referenced and cited by both academia and industry, it is reasonable to believe that Canadian engineering universities and colleges are motivated to report their student enrolment data accurately.

Data on leadership levels within engineering faculty is difficult to determine as nomenclature differs across institutions and across sub-disciplines. As a result, caution should be used when interpreting leadership results within the study. The lack of consistent data on leadership roles will hamper future efforts to determine what level of diversity exists in engineering faculty departmental leadership positions, and if the level of diversity at these levels is changing.

\section{RECOMMENDATIONS:}

Engineering faculties seeking to increase and diversify their enrolment should stress the connection between work within sub-disciplines and the potential to positively change society and the environment. Godwin et al.'s [18] work on how female engineering students articulate a strong career requirement to see their professional work as being able to effect positive social change. The perception that biosystems engineering has a clear link to social wellbeing, may contribute to a higher enrolment rate for women students when compared to sub-disciplines with lower rates, such as software engineering and mechanical.

Recruitment programs and materials could explicitly address how sub-disciplines such as mechanical and software engineering provide career opportunities for exciting technical work and also provide a positive contribution to society, which may attract a more diverse student base and increase the number of women enrolled in these programs.

Future research could investigate how design team diversity impacts design outcomes and examine how heterogeneous worldviews can improve processes and products designed and built by engineers. Such research could make explicit the link between diversity and success within engineering, in education and industry.

This research found that there were distinct and persistent differences between Canadian engineering subdisciplines and schools in the proportion of women students. More research is needed to identify best practices in these sub-disciplines and schools that could potentially shed light on the most effective methods to increase diversity with engineering higher education in Canada.

\section{References}

[1] Othman A. Alijohani, "Comprehensive Review of the Major Studies and Theoretical Models of Student Retention", Higher Education, 6(2), pp.1-18, 2016.

[2] Association of Faculties of Medicine of Canada. "Canadian Medical Education Statistics, 2017, Volume 39", 2088. Available from: https://afmc.ca/sites/default/files/CMES2017-Complete.pdf

[3] Heidi Blackburn," The Status of Women in STEM in Higher Education: A review of the Literature 2007-2017", Science and Technology Libraries, 36:3, pp. 235-273.

[4] J.C. Blickenstaff, "Women and science careers: leaky pipeline or gender filter?" Gender and Education, 17 (4), pp. 369-386, 2005.

[5] Emily Blosser, "Gender Segregation Across Engineering Majors: How Engineering Professors Understand Women's Underrepresentation in Undergraduate Engineering, " Engineering Studies, 9(1), pp. 24-44, 2017.

[6] J. Buolamwini and T. Gebru, "Gender Shades: Intersectional Accuracy Disparities in Commercial Gender Classification," Proceedings of Machine Learning Research 81:1-15, 2018 Conference on Fairness, Accountability, and Transparency, 2018.

[7] Erin Cech, "Culture of Disengagement in Engineering Education?" Science, Technology, \& Human Values, 39 (1) pp. 42-72, 2014. 
[8] Erin Cech, "Engineers and Engineeresses? SelfConceptions and the Development of Gendered Professional Identities." Sociological Perspectives, 58(1), 56-77, 2015

[9] E. Cech and H.M. Sherick, "Depoliticization and the Structure of Engineering Education," in S. Hylgaard Christensen et al. (Eds.), International Perspectives on Engineering Education, pp. 203-216. New York, NY: Springer, 2015.

[10] E. Cech, B. Rubineau, S. Silbey, and C. Serond, "Professional Role Confidence and Gendered Persistence in Engineering," American Sociological Review 76(5), pp. 641-666, 2011

[11] Damon Centola, Joshua Becker, Devon Brackbill \& Andrea Baronchelli, "Experimental evidence for tipping points in social conventions". Science, 360 (6393), pp. 1116-1119, 2018.

[12] Council of Canadian Academies (2015). Some Assembly Required: STEM Skills and Canada's Economic Productivity. The Expert Panel on STEM Skills for the Future. Available from: https://cca-reports.ca/wpcontent/uploads/2018/10/stemfullreporten.pdf

[13] Engineers Canada. Canadian Engineers for Tomorrow. Trends in Engineering Enrolment and Degrees awarded 2006-2010 Report, 2011. Ottawa, Canada.

[14] Engineers Canada. Canadian Engineers for Tomorrow. Trends in Engineering Enrolment and Degrees awarded 2017 Report, 2018. Available from:

https://engineerscanada.ca/reports/enrolment-and-degreesawarded-report

[15] S. Engstrom, "Female Students Who Succeed within Higher Technical Education - When and Why They Choose and Who They Are," Procedia Social and Behavioural Sciences, (167), pp. 161-169, 2015.

[16] N. Ensmenger, "Beards, Beards, Sandals, and Other Signs of Rugged Individualism: Masculine Culture within the Computing Professions," OSIRIS, 30, (1), pp. 38-65, 2015

[17] S. Godec, "Sciencey Girls: Discourses Supporting Working-Class Girls to Identify with Science," Education Sciences, 8(1), pp. 19-36, 2018

[18] Godwin, A, G. Potvin, Z. Hazari, \& J. Lock, "Identity, Critical Agency and Engineering: An Affective Model for Predicting Engineering as a Career Choice," Journal of Engineering Education 105(2), pp. 312-340, 2016

[19] R. Greenfield, Silicon Valley is one big 'ole fraternity. The Wire, April 2012. Available from: https://www.theatlantic.com/technology/archive/2012/04/si licon-valley-big-ole-fraternity/328778
[20] D.H. Hatmaker, Engineering Identity: Gender and Professional Identity Negotiation among Women Engineers. Gender, Work and Organization, 20(4), pp.382396, 2013.

[21] L. Holman, D. Stuart-Fox \& C.E. Hauser, "The gender gap in science: How long until women are equally represented?" PLoS Biol 16(4), 2018.

[22] Kelley, M., \& Bryan, K. (2018) Gendered perceptions of typical engineers across specialties for engineering majors. Gender and Education. 30(1), pp. 22-44.

[23] Marx, D. M., \& Roman, J. S., "Female role models: Protecting female students' math test performance", Personality and Social Psychology Bulletin, 28, pp. 11851197, 2002.

[24] Natalie Mazur, Bronwyn Chorlton, and John Gales, "Comparing the Experiences of Women in Undergraduate Engineering Across Different Schools," Proceedings from the 2019 Canadian Engineering Education Association (CEEA-AECEG19) Conference, 2019.

[25] L. Pacelli, "Asleep at the wheel of auto safety? Recent air bag regulations by the National Highway Traffic Safety Administration," Journal of Contemporary Health Law Policy, 1999, 15, pp.739-77, 1999

[26] Sheryl Sandberg and Neil Scovell, N. Lean in: women, work and the will to lead. New York, NY: Knopf, 2013.

[27] C. Seron, S. Silbey, E. Cech, \& B. Rubineau, "Persistence is Cultural: Professional Socialization and the Reproduction of Sex Segregation," Work and Occupations, 43(2), pp. 178-214, 2016

[28] I.H. Settles, I.H, R. O’Connor \& S.C. Yap, "Climate Perceptions and Identity Interference Among Undergraduate Women in STEM", Psychology of Women Quarterly, (40), pp. 1-38, 2016.

[29] Statistics Canada. Number of full-time teaching staff at Canadian universities, by rank, sex, 2018. Available from: https://www150.statcan.gc.ca/t1/tbl1/en/tv.action?pid=3710 $\underline{007601}$

[30] V. Tinto. Leaving College: Rethinking the Causes and Cures of Student Attrition (2nd ed.). Chicago: University of Chicago Press, 1993.

[31] UNESCO Institute for Statistics. Women in Science. Fact Sheet No. 43, 2015. Available from: http://uis.unesco.org/sites/default/files/documents/fs43women-in-science-2017-en.pdf 